\title{
LOCALLY ADVANCED ESOPHAGEAL CANCER: STATE OF THE ART
}

\section{Authors:}

Elena Asensio Martinez

Alejandra Magdaleno

Maria del Carmen Ors

Maria Ballester

Marta Llopis

Alvaro Javier Arroyo

Javier Gallego Plazas

Affiliations

Filiation: Department of Medical Oncology

Hospital General

Universitario de Elche

(Spain)

\section{Correspondence}

Elena Asensio Martinez

helenasensio@yahoo.es

\section{ABSTRACT}

Esophageal cancer is an important cause of death worldwide, with an increasing incidence in adenocarcinoma subtype (mainly affecting the esophago-gastric junction) in western countries. Nevertheless, squamous cell carcinoma remains the most incident worldwide. An appropriate pretreatment assessment is mandatory to select best treatment in each patient, usually within the consensus of a multidisciplinary tumor board. Esophagogastroduodenoscopy and chest-abdomen CT scan are basic work-up. Although complete surgical resection remains the cornerstone of the treatment for resectable disease, long-term results are poor and recurrences are common, especially in locally advanced setting. Multimodal therapy for locally advanced disease improves survival nevertheless; the optimal therapeutic approach remains controversial. Preoperative chemoradiotherapy and perioperative chemotherapy are the most common strategies. However, in some patients, definitive chemoradiotherapy without surgery is also an option. In this review, we will cover the epidemiology, diagnosis and staging of patients with esophageal cancer. We will also discuss the main clinical trials and meta-analysis in the treatment of local and locally advanced esophageal cancer, evidence for different multimodal approaches (with and without surgery) and finally we will propose a treatment algorithm.

Key words: esophageal cancer, esophago-gastric junction cancer, chemotherapy, radiation, surgery, multimodal therapy, algorithm. 


\section{INTRODUCTION}

Esophageal cancer (EC) is the 6th most common cause of death from cancer and the 8th leading cancer in the world. All over the world, 455800 new cases were diagnosed and 400200 deaths occurred in 2012 (1).

EC has two main subtypes, squamous cell carcinoma (SCC) and adenocarcinoma (ADC). Small cell carcinoma and sarcomas arising in the esophagus and esophago-gastric junction (EGJ) are rare entities. Borderline location of EGJ cancers has caused their inclusion in both, esophageal and gastric cancer studies. Tumors arising from the distal 5 $\mathrm{cm}$ of the esophagus are considered Siewert type I, those arising from de EGJ are Siewert type II and those from the cardia of the stomach (within $5 \mathrm{~cm}$ of the EGJ) with extension into EGJ or esophagus are Siewert type III. (2)

The incidence of EC varies between different areas worldwide. The highest rates are found in Southern Africa and East of Asia, with $90 \%$ corresponding to SCC. SCC predominates in the upper and mid-esophagus and is associated with smoking and alcohol habits; esophageal squamous dysplasia is the precursor lesion. In western countries, SCC has been decreasing because of the reduction in tobacco and alcohol consumption. On the other hand, ADC occurs predominantly in the lower esophagus and in the EGJ. The incidence rates of ADC have been increasing dramatically in western countries in the last years, mostly due to the increase of overweight and obesity which are associated with gastric reflux and the precursor state Barrett's esophagus (3).

Both subtypes of EC have similar clinical manifestations. The most common symptom of EC is dysphagia, typically accompanied by weight loss; restrosternal discomfort and anemia are also frequent.
Less common symptoms are cough or lung infections. However, an increasing number of asymptomatic cases are being discovered, usually in patients with Barrett's esophagus as part of surveillance endoscopy program (4).

Beyond histopathological and epidemiologic differences, molecular features are also distinct in SCC and ADC. A recent publication (5) showed that SCC is molecularly a more reminiscent disease to other squamous carcinomas of other organs. ADC seems more to CIN (chromosomal instability) gastric cancer. Three different subclasses of SCC were found, with frequent amplifications of CCND1 and SOX2 and/or TP63. On the other hand, ERBB2, VEGFA, GATA 4 and GATA 6 were more commonly amplified in ADC. HER2-neu amplification and overexpression is also implicated in some $\mathrm{EC}$ and EGJ cancers, mainly in ADC, being immunohistochemistry the most widely used test for assessment. Nowadays, amplification of HER2 only has therapeutic implications in advanced disease with the indication for use use of trastuzumab added to chemotherapy.

\section{DIAGNOSIS AND STAGING}

Esophagogastroduodenoscopy is required to obtain biopsy samples to confirm the diagnosis and histological subtype as well as to know the mucosal extension of the disease. The staging work-up should include chest-abdomen CT scan. In case of no evidence of metastatic spread, we should complete staging performing an endoscopic ultrasonography and PET and bronchoscopy when needed. Endoscopic ultrasonography further improves assessment of tumor and lymph-node status, and may provide pathologic evaluation of lymph nodes with the use of fine-needle aspiration. PET scan may identify occult distant metastatic disease, 


\section{LOCALLY ADVANCED ESOPHAGEAL CANCER: STATE OF THE ART}

overstaging to stage IV in up to $10-20 \%$ of patients. Bronchoscopy is necessary in SCC, mainly in tumors located in upper or mid esophagus where bronchial tree could be involved. It is also recommended to evaluate patient comorbidities in order to define if is fitness for surgery $(3,4)$.

Patients with EC (including EGJ cancer) should be staged according to UICCAJCC TNM staging system ( $8^{\text {th }}$ edition). It is important to remark that prognostic groups/anatomic stages are different for $\mathrm{ADC}$ or SCC (6).

\section{TREATMENT}

\subsection{GENERAL CONSIDERATIONS}

We define three different groups in EC with treatment implications: local EC (T1-T2 N0), locally advanced EC (T3-T4 or N1-N3) and metastatic (any T, any N, M1).

In patients with adenocarcinoma T1a and less than $2 \mathrm{~cm}$ in diameter, endoscopic mucosal resection is the preferred approach with a risk of lymph-node metastasis around $1-2 \%$. In patients with T1b tumors, the risk of lymph-node metastasis is $20 \%$, so radical esophagectomy is the preferred treatment. In T2 tumors radical surgery is considered the standard treatment.

Treatment of metastatic and recurrent EC has two main settings: a) palliative chemotherapy based on cisplatin or oxaliplatin combined with infusional 5FU or capecitabine (a third drug may improve response rates but is associated with more toxicity) with the aims of improving survival and quality of life; b) local therapies such as endoscopic placement of stents, endoscopic dilation or radiotherapy to palliate obstructive symptoms.

In this review, we will focus on the treatment of locally advanced EC (cT3T4 or cN1-N3).

\subsection{LOCALLY ADVANCED EC}

In this group of patients with locally advanced EC, surgery alone is not a standard treatment due to difficulties on achieving complete resection and poor results. Between 30 to $50 \%$ of cases do not achieve complete tumor resection (R0), and even after R0 surgery, relapse (local or metastatic) is common, with long-term survival around 20\% (7). This fact prompted clinical research in locally advanced EC with major evolution in the last 15 years. Multimodality treatment, in addition to surgery with radiotherapy and/or chemotherapy (to control micrometastatic disease and to improve radiation effects) has been widely investigated in different settings:

- Preoperative and perioperative chemotherapy

- Preoperative chemoradiotherapy

- Definitive chemoradiotherapy

\section{Preoperative and perioperative chemotherapy}

Distant recurrence following curative resection is an important problem in localized EC that limits survival. The presence of micrometastasis that leads to failure in curatively treated patients guided the investigation to explore the role of induction preoperative chemotherapy. The aim of preoperative chemotherapy is exterminating micrometastasis, downstage the tumor enhancing resectability, improve locoregional control and finally improve overall survival (8). But on the other hand, it exists the risk of progression disease and compromising definitive treatment.

The use of chemotherapy followed by surgery has been studied since late 1970s and was evaluated in parallel with preoperative chemoradiotherapy and definitive chemoradiotherapy trials. 


\section{LOCALLY ADVANCED ESOPHAGEAL CANCER: STATE OF THE ART}

After some small trials with few data, the Radiation Therapy Oncology Group trial 8911 (USA Intergroup 113) randomized 467 patients with EC (51\% ADC, 44\% SCC and 5\% unclassified) to induction chemotherapy (three cycles of cisplatin and 5-fluorouracil (5-FU)) followed by surgical resection or surgery alone (9). After R0 resection, patients in experimental arm received also two cycles of chemotherapy although only $52 \%$ of those started it, and only $38 \%$ completed all planned cycles. The primary end-point was overall survival. No difference was observed between the control group and the experimental arm in terms of overall survival (16.1 vs 14.9 months, $\mathrm{p}=0.53$ ), 3 -year survival (26 vs 23\%, HR 1.04 (95\% CI 0.84 - 1.29), $\mathrm{p}=0.65), \mathrm{R} 0$ resection rate (59 vs $62 \%$ ) and treatment related mortality (6 vs $7 \%$, $\mathrm{p}=0.33$ ). Long term results of this trial were published in 2007, confirming absence of statistically significant differences between both arms of treatment (10).

In contrast to the results of this clinical trial, the Medical Research Council Oesophageal Cancer Working Group developed in the early 1990s the OEO2 trial. 802 patients with resectable EC (31\% SCC and 69\% ADC) were randomized to one of two arms: 2 cycles of chemotherapy (cisplatin and continuous infusion for 4 days of 5-FU) followed by surgery or resection alone. Patients assigned to experimental arm had statistically significant better overall survival (HR 0.79 (95\% CI 0.67 - 0.93), $\mathrm{p}=0.004)$ as well as better $\mathrm{R} 0$ resection rate $(60 \%$ vs $54 \%, \mathrm{p}<0.0001)$ with the same mortality rate in both treatment groups (10\%) (11). Long-term follow-up confirmed the results, with a 5-year survival rate of 23 vs $17.1 \%$ (HR 0.84 (95\% CI 0.72 - 0.98), $\mathrm{p}=0.03$ ) (12).

The discordance between the results of these two trials inspired a wide debate.
The differences in results could be explained by larger sample size in OEO2 trial and duration of preoperative chemotherapy (shorter in OEO2 trial) that could have decreased the risk of disease progression during preoperative treatment.

In the MAGIC trial, carried out by the British Medical Research Council, 503 patients with gastric (75\%), distal esophageal $(14 \%)$ or EGJ $(11 \%)$ adenocarcinoma were assigned to receive three cycles of preoperative chemotherapy based in epirubicin, cisplatin and 5-FU, and three cycles of the same chemotherapy postoperative or surgery alone (13). The primary endpoint was overall survival and it was met, with a 36 vs $23 \%$ five-year survival rate (HR 0.75 (95\% CI $0.60-0.93), \mathrm{p}=0.009$ ). $91 \%$ of patients in perioperative chemotherapy arm underwent surgery, whereas $96.4 \%$ in the surgery group did so. Despite improvement in overall survival with perioperative chemotherapy, there was no improvement in $\mathrm{R} 0$ resection rate (66 vs $69 \%$ ). The incidence of postoperative complications was similar in the two groups (45.7 vs $45.3 \%$ ) as also were the number of deaths within 30 days after surgery (5.6 vs $5.9 \%$ ). Only $41.6 \%$ of the patients in the experimental arm could complete all six cycles of chemotherapy, mainly due to disease progression or early death, patient choice and postoperative complications.

Similar to the MAGIC trial, the FNCLCC/FFCD trial randomized 224 patients with resectable adenocarcinoma of the distal esophagus (11\%), EGJ $(64 \%)$ or stomach $(25 \%)$ to perioperative chemotherapy (two or three preoperative cycles of infusional 5-FU and cisplatin every four weeks and three or four postoperative cycles, for a total of six cycles) or surgery alone (14). Perioperative chemotherapy improved the R0 resection rates (84 vs $73 \%, p=0.04$ ), 


\section{LOCALLY ADVANCED ESOPHAGEAL CANCER: STATE OF THE ART}

5 -year overall survival rate (38 vs $24 \%$, HR 0.69 (95\% CI $0.50-0.95), \mathrm{p}=0.02)$ and 5-year disease free survival rate $(34$ vs $19 \%$, HR 0.65 (95\% CI $0.48-0.89)$, $\mathrm{p}=0.003$ ). Postoperative complications and postoperative deaths were similar in the two groups. As it was the case for the MAGIC trial, only $50 \%$ of patients in the experimental arm completed postoperative chemotherapy.

The role of preoperative/perioperative chemotherapy has been addressed in many other trials, and pooled in some meta-analysis. A total of 10 randomized trials involving 2122 patients with EC were included in a meta-analysis published by Cochrane (15). The results showed an improvement in overall survival for patients who received preoperative chemotherapy (HR 0.88, (95\% CI $0.80-0.96), \mathrm{p}=0.003)$ and, also a higher rate of $\mathrm{R} 0$ resection (RR 1.11 (95\% CI 1.03 - 1.19). No differences were found in overall resection rate, tumor recurrence or nonfatal complications. Another meta-analysis published in 2011 (16) evaluated twentyfour clinical trials, nine of them were randomized comparisons of neoadjuvant chemotherapy versus surgery alone, with 1981 patients included. The HR for allcause mortality for neoadjuvant chemotherapy was 0.87 (95\% CI 0.79 $0.96, \mathrm{p}=0.005)$, for SCC it was only 0.92 (95\% CI $0.81-1.04, \mathrm{p}=0.18)$ and 0.83 $(95 \%$ CI $0.71-0.95, \mathrm{p}=0.01)$ for ADC.

\section{Preoperative chemoradiotherapy}

Initial trials explored the role of preoperative radiotherapy in order to improve loco-regional control with disappointing results. Thus, the failure of radiotherapy in this preoperative setting led to the integration of chemotherapy and radiotherapy in the same scenario. Radiotherapy was expected to improve loco-regional control and chemotherapy to eradicate micrometastasis in addition to its radiosensitazing effect. The most important trials in this setting are:

In the CROSS trial carried out between 2004 and 2008, 366 patients (75\% ADC, $23 \%$ SCC and $2 \%$ undifferentiated) with EC or EGJ cancer were randomized to chemoradiotherapy followed by surgery or surgery alone (17). Patients in chemoradiotherapy arm received weekly carboplatin and paclitaxel for 5 weeks concurrently with radiotherapy (41.4 Gy in 23 fractions). $\mathrm{R} 0$ resection rates were better in the experimental compared to the control arm, 92 vs $69 \%, \mathrm{p}<0.001)$ and $29 \%$ of patients achieved pathological complete response in the chemoradiotherapy arm. Postoperative complications and mortality rates were similar in the two arms. Long-term results (18) after a median follow up of 84.1 months showed a statistically significant survival advantage for preoperative chemoradiotherapy with a median overall survival of 48.6 months in the experimental arm and 24 months in the surgery group (HR 0.68, $(95 \% \mathrm{CI}$ 0.53 - 0.88), $\quad \mathrm{p}=0.003)$. Survival improvement was higher for squamous carcinomas (81.6 vs 21.1 months (HR $0.48(95 \%$ CI $0.28-0.83), \mathrm{p}=0.008)$ than for adenocarcinoma (43.2 vs 27.1 months (HR 0.73 (95\% CI $0.55-0.98), \mathrm{p}=0.038$ ). In contrast to the results of CROSS trial, the French group published the results of the FFCD 9901 trial. This trial (19) was conducted between 2000 and 2009 in patients with stage I or II EC $(70.3 \%$ SCC, $29.2 \% \quad \mathrm{ADC}$ and $0.5 \%$ undifferentiated carcinoma). 195 patients were randomized to preoperative chemoradiotherapy followed by surgery or surgery alone. Patients received $45 \mathrm{~Gy}$ in 25 fractions and two cycles of 5-FU and cisplatin in the experimental arm. The results showed no statistically significant differences in $\mathrm{R} 0$ resection rates $(93.8 \%$ in the experimental arm vs $92.1 \%$ in control arm, $\mathrm{p}=0.749$ ), and no 


\section{LOCALLY ADVANCED ESOPHAGEAL CANCER: STATE OF THE ART}

improvement in 3-year OS (47.5 vs 53\%, HR 0.99 (95\% CI 0.69 - 1.40), $\mathrm{p}=0.94)$. Mortality was even higher in patients receiving preoperative chemoradiotherapy $\quad(11.1$ vs $3.4 \%$, $\mathrm{p}=0.049$ ).

Discrepancy in outcomes of this two trials could be explained by: a) smaller sample size in the FFCD 9901 trial; b) different proportion of histological subtypes between the two studies; c) more patients with earlier stage disease in the FFCD 9901 trial; d) less toxic chemoradiotherapy regimen administered in the CROSS trial.

At meta-analysis level (16), with twelve randomized clinical trials comparing neoadjuvant chemoradioterapy vs surgery alone including 1854 patients, HR for allcause mortality for neoadjuvant chemoradiotherapy was $0.78(95 \% \mathrm{CI}$ $0.70-0.88, \mathrm{p}<0.0001)$; HR for SCC only was $0.80(95 \%$ CI $0.68-0.93, p=0.004)$ and it was $0.75(95 \%$ CI $0.59-0.95$, $\mathrm{p}=0.02$ ) for $\mathrm{ADC}$.

Considering the results of MAGIC and CROSS trials, patients could benefit of two different approaches in the preoperative setting. The maximum benefit in CROSS trial was observed in SCC, but it was consistent across subgroups. Two small randomized trials in patients with ADC did not show significant differences in survival between preoperative chemoradiotherapy and preoperative chemotherapy $(20,21)$. More recently, some trials have investigated this issue. In the Neo-RES trial (22), 181 patients in Norway and Sweden with EC and EGJ cancer were randomized to three cycles of chemotherapy (cisplatin and 5-FU) with or without radiotherapy (40 Gy) followed by surgery. Primary end-point was histological complete response. Results showed a better histological complete response in the chemoradiotherapy group (28 vs 9\%, p=0.002). R0 resection rate was also better in the chemoradiotherapy arm $(87 \%$ vs $74 \%, \quad \mathrm{p}=0.04)$. No differences were found in overall survival.

More recently, a retrospective multicenter European study was published, comparing survival from neoadjuvant chemotherapy vs neoadjuvant chemoradiotherapy in patients with ADC (23). Between 2001 and 2012, 608 patients were included from 10 European centers. There were no statistically significant differences between the two arms neither in 3-year overall survival (57.9 vs 53.4\%, HR 0.89 (95\% CI 0.67 1.17), $\mathrm{p}=0.391)$ nor in disease-free survival (52.9 vs $48.9 \%$, HR 0.90 (95\% CI $0.69-1.18), \mathrm{p}=0.443)$.

Maybe Neo-AEGIS trial (24), which pretends to include 574 patients with esophageal ADC for randomization between CROSS or MAGIC regimens, will help us to decide about the best strategy. However, results are not expected before 2021. Nowadays, the practice varies worldwide: perioperative chemotherapy is considered the standard treatment in most European countries, whereas neoadjuvant chemoradiotherapy is commonly used in United States.

Initial results of CALGB 80803 have been recently reported (25). This phase 2 trial evaluates the use of early assessment of chemotherapy responsiveness by PET imaging to guide further therapy in patients with EC and EGJ cancer. 257 patients were randomized to 3 cycles of FOLFOX or 2 cycles of carboplatin and paclitaxel, and PET scan was performed before starting chemotherapy and after completing induction treatment. PET responders continued with the same chemotherapy concurrently with radiotherapy and non-responders changed to the other chemotherapy regime concurrently with radiotherapy. In this trial, radiotherapy dose was $50 \mathrm{~Gy}$. The results showed $18 \%$ pathological 


\section{LOCALLY ADVANCED ESOPHAGEAL CANCER: STATE OF THE ART}

complete response rate in all nonresponders and $26 \%$ in responders. In FOLFOX arm, responding patients achieved pathological complete response in $37.5 \%$ of patients. PET scan could be incorporated in future trials in order to identify more effective treatments and FOLFOX and 50 Gy dose radiotherapy should be evaluated in a phase III trial.

Given the low rate of pathological complete response in patients treated with chemoradiotherapy followed by surgery new adjuvant therapies are being explored. Nivolumab is a fully human monoclonal antibody that targets PD-1 (programmed death-1, an inmunoinhibitory receptor that regulates $\mathrm{T}$-cell activation). Nivolumab has shown promising activity in phase I and phase II in metastatic EC. CheckMate 577 is a phase 3, double-blind, multinational trial, for patients with stage II/III EC or EGJ cancer who complete preoperative chemoradiotherapy followed by surgery and evidence of residual pathologic disease. In this trial, patients are randomized to nivolumab or placebo (26). The estimation of patients to include is 760. Primary end points are diseasefree survival and overall survival and estimated study completion date is april 2021.

\section{Definitive chemoradiotherapy}

Non-surgical approach for EC arised because of poor long-term results with surgery, specially, in patients with unresectable tumors, serious comorbidities or old age.

The population selected for nonsurgical approach is different for several reasons: a) patients with unfavorable prognostic features (as medical contraindications and primary unresectable); b) reports of nonsurgical therapy are based on clinical staging, being less accurate than pathological staging obtained after surgical treatment; c) some patients treated without surgery are treated with a more palliative than curative intention, receiving less intense treatment (chemo and radiotherapy).

After disappointing results during the 1970s for SCC treated with surgery or radiotherapy alone, improvements in radiotherapy techniques and advent of new chemotherapeutic agents made the Radiation Therapy Oncology Group (RTOG) to launch a prospective, randomized, phase III clinical trial in 1985 to test whether chemoradiotherapy followed by chemotherapy could improve the overall survival rate in patients with thoracicus EC compared to RT alone (27). Initially, only patients with SCC were included, but since 1986 also patients with ADC were randomized. Between 1985 and 1990, 129 patients were ranzomized to receive radiotherapy alone (64 Gy in 32 fractions) or chemoradiotherapy (cisplatin and infusional 5-FU every three weeks for four cycles combined with $50 \mathrm{~Gy}$ of radiotherapy in 25 fractions). After a median follow-up of 5 years, overall survival rate was 26\% (95\% IC 15-37\%) for the combined treatment arm compared to $0 \%$ in patients receiving only radiotherapy. Chemotherapy could be administered as planned only in $68 \%$ of patients. Based on the results of this trial, the standard therapy for patients with EC selected for nonsurgical treatment became chemoradiotherapy. In order to improve these results, INT 0122 trial was designed. In INT 0122 trial chemotherapy and radiotherapy were both intensified: a) number of chemotherapy cycles was increased from four to five; b) 5-FU infusion was longer, with one day more of treatment; c) three cycles of chemotherapy were administered before the combined treatment; d) total dose of radiotherapy was increased from 50 to $64.8 \mathrm{~Gy}$. But results of this trial were disappointing: 


\section{LOCALLY ADVANCED ESOPHAGEAL CANCER: STATE OF THE ART}

survival and local control rates were similar to the ones in RTOG 85-01 trial, however toxicity was higher, with treatment-related mortality rate of $9 \%$ vs $2 \%(28,29)$. Consequently, RTOG 94-05 trial, with 236 patients, compared four monthly cycles of chemotherapy (infusional 5-FU (4 days) and cisplatin) with either 50.4 Gy or 64.8 Gy of concurrent radiation therapy (30). Trial was prematurely closed after an interim analysis with 16.4 months of follow up, which showed no differences in overall survival and locoregional control but higher mortality (eleven deaths vs two) in the high-dose radiation therapy arm. Considering the results of these two last mentioned trials, standard treatment for nonsurgical patients with EC remained chemoradiotherapy with 5-FU and cisplatin, and 50.4 Gy radiation.

Nevertheless, cisplatin is difficult to administer in some patients because of the long intravenous hydration needed and oxaliplatin could be a better option for them; also the addition of leucovorin to modulate 5-FU could improve the results. The combination of oxaliplatin and 5-FU with leucovorin (FOLFOX) was tested in the PRODIGE5/ACCORD17 clinical trial (31). This phase II/III trial was conducted between 2004 and 2011 in France and 267 patients were randomized to six cycles of FOLFOX (three concurrently with radiotherapy) or four cycles of cisplatin and 5-FU (two concurrently with radiotherapy). Both arms received 50 Gy of radiotherapy in 25 fractions. After a median follow-up of 25.3 months, median progression-free survival was 9.7 months in FOLFOX group and 9.4 in control arm (HR 0.93 (95\% CI 0.70 1.24), $\mathrm{p}=0.64)$. No significant differences were found in grade 3 or 4 adverse events. Although this trial did not meet its primary end-point, definitive chemoradiotherapy with FOLFOX emerged as an option for patients with problems to tolerate cisplatin and its hydration.

Furthermore, considering results from nonrandomized trials where patients were treated with chemoradiotherapy with or without surgery, FFCD group developed the 9201 trial (32). This trial compared the results of chemoradiotherapy alone or followed by surgery in responding patients. 444 patients were included and received chemoradiotherapy (2 cycles of cisplatin and 5-FU with a radiotherapy dose of 46 Gy). 259 responding patients were randomized to surgery or continuation of chemoradiation (3 cycles more of the same chemotherapy and 20 Gy more of radiation). $88.8 \%$ of patients randomized had SCC and $11.2 \%$ ADC. Results showed 17.7 months median survival in surgery arm vs 19.3 in chemoradiotherapy alone arm (HR 0.88, $\mathrm{p}=0.44)$. Three-months mortality rate was $9.3 \%$ in surgery vs $0.8 \%$ in chemoradiotherapy group $(\mathrm{p}=0.02)$. Investigators suggested there was no benefit with the addition of surgery in SCC patients who responded to chemoradiation.

\subsection{TREATMENT ALGORITHM}

After covering main clinical trials in EC we propose a treatment algorithm.

In patients with locally advanced disease, in case of unfit patient for surgery but fit enough for chemotherapy and radiotherapy, definitive chemoradiotherapy may be the preferred option. Chemoradiotherapy is also the treatment of choice in cervical EC. For patients fit enough for surgery, neoadjuvant chemoradiotherapy seems to be the standard treatment for SCC, while either neoadjuvant chemoradiotherapy of perioperative chemotherapy would be recommended for ADC (this last option specially for patients with EGJ tumors) (figure 1). 


\section{LOCALLY ADVANCED ESOPHAGEAL CANCER: STATE OF THE ART}

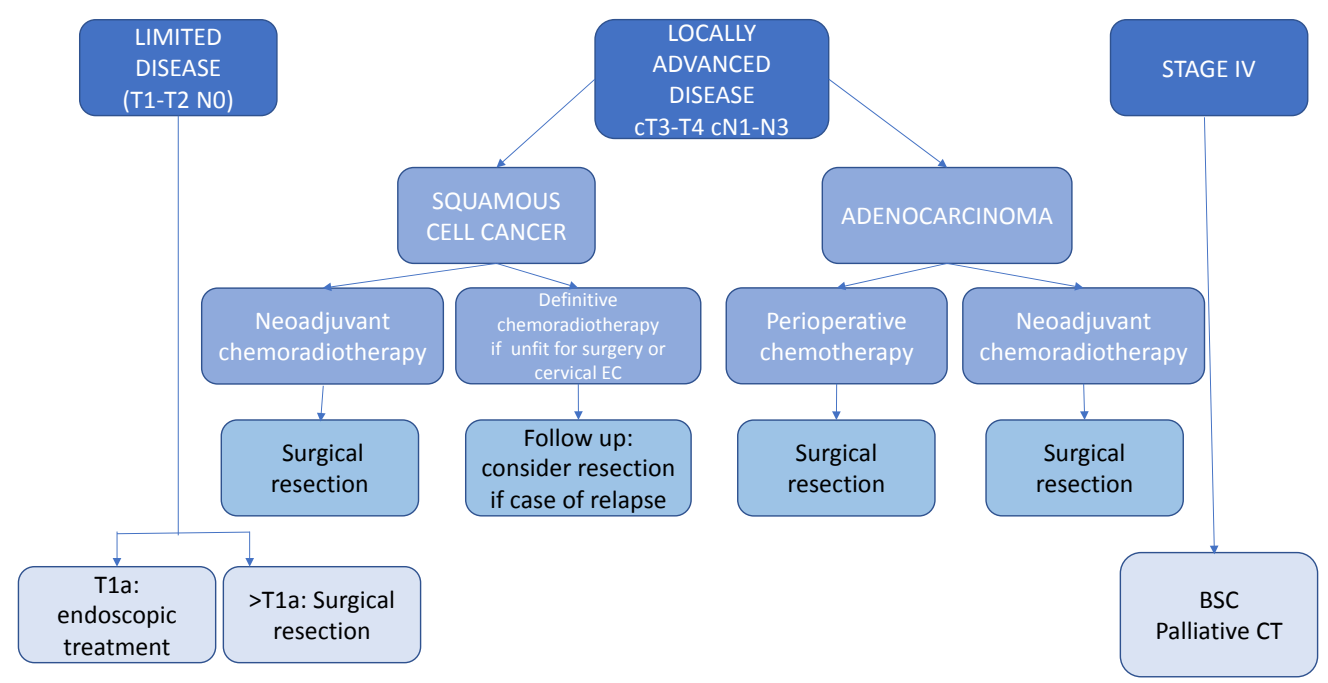

Figure 1.-

\section{CONCLUSIONS AND FUTURE PERSPECTIVES}

In summary, surgery remains the cornerstone for resectable EC and EGJ tumors. However, long-term outcomes for surgery alone are poor. Neoadjuvant therapy (chemotherapy alone or chemoradiotherapy), is considered standard for fit for surgery patients and definitive chemoradiotherapy for those unfit. Based on current available data we can conclude that preoperative therapies offer better R0 resection rate as well as survival improvements. Best therapeutic choice should be decided within a multidisciplinary tumor board. Nevertheless, even taking into account improvements in outcomes with current strategies, further research is needed incorporating growing knowledge in the molecular field to clinical trials so that we can broaden perspectives to our patients. 


\section{REFERENCES}

1. Torre LA, Bray F, Siegel RL, et al. Global cancer statistics, 2012. CA Cancer J Clin 2015; 65:87.

2. Ashraf N, Hoffe S, Kim R. Locally advanced gastroesophageal junction tumor: a treatment dilemma. Oncologist. 2015 Feb;20(2):134-42. doi: 10.1634/theoncologist.2014-0377.

3. Rustgi A, El-Serag HB. Esophageal carcinoma. N Engl J Med. 2015 Apr 9;372(15):1472-3. doi: 10.1056/NEJMc1500692

4. Pennathur A, Gibson MK, Jobe BA, Luketich JD. Oesophageal carcinoma. Lancet. 2013 Feb 2;381(9864):400-12. doi: 10.1016/S0140-6736(12)60643-6.

5. The Cancer Genome Atlas Research Network. Integrated genomic characterization of oesophageal carcinoma. Nature. 2017 Jan 12;541(7636):169-175. doi: 10.1038/nature20805

6. Rice TW, Kelsen DP, Blackstone $\mathrm{EH}$, et al. Esophagus and esophagogastric junction. In: Amin MB, Edge SB, Greene FL, et al., eds. AJCC Cancer Staging Manual. 8th ed. New York, NY: Springer; 2017:185-202.

7. Reed CE. Surgical management of esophageal carcinoma. Oncologist. 1999;4(2):95-105

8. Garg PK, Sharma J, Jakhetiya A, Goel A, Gaur MK. Preoperative therapy in locally advanced esophageal cancer.

World J Gastroenterol. 2016 Oct 21;22(39):8750-8759.

9. Kelsen DP, Ginsberg R, Pajak TF, Sheahan DG, Gunderson L, Mortimer J,et al. Chemotherapy followed by surgery compared with surgery alone for localized esophageal cancer. N Engl J Med. 1998 Dec 31;339(27):1979-84.

10. Kelsen DP, Winter KA, Gunderson LL, Mortimer J, Estes NC, Haller DG,et al; Radiation Therapy Oncology Group.; USA Intergroup. Long-term results of RTOG trial 8911 (USA Intergroup 113): a random assignment trial comparison of chemotherapy followed by surgery compared with surgery alone for esophageal cancer. J Clin Oncol. 2007 Aug 20;25(24):3719-25.

11. Medical Research Council Oesophageal Cancer Working Party: Surgical resection with or without preoperative chemotherapy in oesophageal cancer: A randomised controlled trial. Lancet 359: 727-1733, 2002

12. Allum WH, Stenning SP, Bancewicz J, Clark PI, Langley RE. Long-term results of a randomized trial of surgery with or without preoperative chemotherapy in esophageal cancer. J Clin Oncol. 2009 Oct 20;27(30):5062-7. doi: 10.1200/JCO.2009.22.2083.

13. Cunningham D, Allum WH, Stenning SP, Thompson JN, Van de Velde CJ, Nicolson M, Scarffe JH, Lofts FJ, Falk SJ, Iveson TJ, Smith DB, Langley RE, Verma M, Weeden S, Chua YJ, MAGIC Trial Participants.

Perioperative chemotherapy versus surgery alone for resectable gastroesophageal cancer. N Engl J Med. 2006 Jul 6;355(1):11-20.

14. Ychou M, Boige V, Pignon JP, Conroy $\mathrm{T}$, Bouché $\mathrm{O}$, Lebreton 


\section{LOCALLY ADVANCED ESOPHAGEAL CANCER: STATE OF THE ART}

G,et al. Perioperative chemotherapy compared with surgery alone for resectable gastroesophageal adenocarcinoma: an FNCLCC and FFCD multicenter phase III trial. J Clin Oncol. 2011 May 1;29(13):171521. doi:

10.1200/JCO.2010.33.0597.

15. Kidane B, Coughlin S, Vogt K, Malthaner R. Preoperative chemotherapy for resectable thoracic esophageal cancer. Cochrane Database Syst Rev. 2015 May 19;(5):CD001556. doi: 10.1002/14651858.CD001556.pub 3.

16. Sjoquist KM, Burmeister BH, Smithers BM, Zalcberg JR, SimesRJ, Barbour A, Gebski V; Australasian Gastro-Intestinal Trials Group. Survival after neoadjuvant chemotherapy or chemoradiotherapy for resectable oesophageal carcinoma: an updated meta-analysis. Lancet Oncol. 2011 Jul;12(7):681-92. doi: 10.1016/S1470-2045(11)70142-5.

17. van Hagen $P$, Hulshof MC, van Lanschot JJ, Steyerberg EW, van Berge Henegouwen MI, Wijnhoven BP, et al; CROSS Group. Preoperative chemoradiotherapy for esophageal or junctional cancer. N Engl J Med. 2012 May 31;366(22):207484. doi: 10.1056/NEJMoa1112088.

18. Shapiro J, van Lanschot JJ, Hulshof MC, van Hagen P, van Berge Henegouwen MI, Wijnhoven BP, van Laarhoven HW, et al; CROSS study group. Neoadjuvant chemoradiotherapy plus surgery versus surgery alone for oesophageal or junctional cancer (CROSS): long-term results of a randomised controlled trial.
Lancet Oncol. 2015

Sep;16(9):1090-8. doi: 10.1016/S1470-2045(15)00040-6.

19. Mariette C, Dahan L, Mornex F, Maillard E, Thomas PA, Meunier $B$, et alF. Surgery alone versus chemoradiotherapy followed by surgery for stage I and II esophageal cancer: final analysis of randomized controlled phase III trial FFCD 9901. J Clin Oncol. 2014 Aug 10;32(23):2416-22. doi: 10.1200/JCO.2013.53.6532.

20. Burmeister BH, Thomas JM, Burmeister EA et al. Is concurrent radiation therapy required in patients receiving preoperative chemotherapy for adenocarcinoma of the oesophagus? A randomized phase II trial. Eur J Cancer 2011; 47: 354-360.

21. Stahl M, Walz MK, Stuschke M, Lehmann N, Meyer HJ, RieraKnorrenschild J, et al. Phase III comparison of preoperative chemotherapy compared with chemoradiotherapy in patients with locally advancedadenocarcinoma of the esophagogastric junction. J Clin Oncol. 2009 Feb 20;27(6):851-6. doi: 10.1200/JCO.2008.17.0506.

22. Klevebro F, Alexandersson von Döbeln G, Wang N, Johnsen G, Jacobsen AB, Friesland S, et al. A randomized clinical trial of neoadjuvant chemotherapy versus neoadjuvant chemoradiotherapy for cancer of the oesophagus or gastro-oesophageal junction. Ann Oncol. 2016 Apr;27(4):660-7. doi: 10.1093/annonc/mdw010.

23. Markar SR, Noordman BJ, Mackenzie H, Findlay JM, Boshier $\mathrm{PR}$, Ni M, et al. Multimodality treatment for esophageal adenocaricnoma: multi-center 


\section{LOCALLY ADVANCED ESOPHAGEAL CANCER: STATE OF THE ART}

propensity-score matched study. Ann Oncol. 2016 Oct 25. pii: mdw560. doi: 10.1093/annonc/mdw560.

24. Reynolds JV. Randomised clinical trial of neoadjuvant and adjuvant chemotherapy (MAGIC Regimen) vs. neoadjuvant chemoradiation (CROSS protocol) in adenocarcinoma of the oesophagus and oesophago-gastric junction. https://clinicaltrials.gov/ct2/show/ NCT01726452

25. Goodman KA, Niedzwiecki D, Hall N, Bekaii-SaabTS, Ye X, Meyers MO, et al. Initial results of CALGB 80803 (Alliance): A randomized phase II trial of PET scandirected combined modality therapy for esophageal cancer. J Clin Oncol 35, 2017 (suppl 4S; abstract 1)

26. Kelly RJ, Lockhart AC, Jonker DJ, Melichar B, Andre T, Chau I, et al. CheckMate 577: A randomized, double-blind, phase 3 study of nivolumab (Nivo) or placebo in patients (Pts) with resected lower esophageal (E) or gastroesophageal junction (GEJ) cancer. J Clin Oncol 35, 2017 (suppl 4S; abstract TPS212)

27. Cooper JS, Guo MD, Herskovic A, Macdonald JS, Martenson JA Jr, Al-Sarraf M, et al. Chemoradiotherapy of locally advanced esophageal cancer: long-term follow-up of a prospective randomized trial (RTOG 85-01). Radiation Therapy Oncology Group. JAMA. 1999 May 5;281(17):1623-7.

28. Minsky BD, Neuberg D, Kelsen $\mathrm{D}$, et al: Neoadjuvant chemotherapy plus concurrent chemotherapy and high dose radiation for squamous cell carcinoma of the esophagus: A preliminary analysis of the phase II intergroup trial 0122. J Clin Oncol 14:149-155, 1996

29. Minsky BD, Neuberg D, Kelsen $\mathrm{DP}$, et al: Final report of intergroup trial 0122 (ECOG PE289, RTOG 90-12): Phase II trial of neoadjuvant chemotherapy plus concurrent chemotherapy and highdose radiation for squamous cell carcinoma of the esophagus. Int J Radiat Oncol Biol Phys 43:517-523, 1999

30. Minsky BD, Pajak TF, Ginsberg RJ, Pisansky TM, Martenson J, Komaki R, et al. INT 0123 (Radiation Therapy Oncology Group 94-05) phase III trial of combined-modality therapy for esophageal cancer: high-dose versus standard-dose radiation therapy.J Clin Oncol. 2002 Mar 1;20(5):1167-74.

31. Conroy T, Galais MP, Raoul JL, Bouché O, Gourgou-Bourgade S, Douillard JY, et al. Definitive chemoradiotherapy with FOLFOX versus fluorouracil and cisplatin in patients with oesophageal cancer (PRODIGE5/ACCORD17): final results of a randomised, phase $2 / 3$ trial. Lancet Oncol. 2014 Mar;15(3):305-14. doi: 10.1016/S1470-2045(14)70028-2.

32. Bedenne L, Michel P, Bouché O, Milan C, Mariette C, Conroy T, et al. Chemoradiation followed by surgery compared with chemoradiation alone in squamous cancer of the esophagus: FFCD 9102. J Clin Oncol. 2007 Apr 1;25(10):1160-8. 
Medical Research Archives. Volume 5, issue 4. April 2017.

LOCALLY ADVANCED ESOPHAGEAL CANCER: STATE OF THE ART 